\title{
Decreased vaccine protection of egg-based influenza vaccine in the elderly and nonhemagglutinin-focused immunity
}

\author{
Karen J. Gonzalez ${ }^{1}$ and Eva M. Strauch ${ }^{1,2}$ \\ IInstitute of Bioinformatics and 2Department of Pharmaceutical and Biomedical Sciences, University of Georgia, Athens, Georgia, USA.
}

\begin{abstract}
Severe influenza illness or death is a serious concern among the elderly population despite vaccination. To investigate how the adaptive immune response after vaccination varies with the patient's age, Jung et al., in a recent issue of the $J C l$, extensively analyzed the serum antibody response in different age groups after immunization with the egg-based influenza vaccine Fluzone. As expected, the immune response in young adults was dominated by antibodies targeting the influenza hemagglutinin (HA) protein. On the contrary, the serological repertoire of elderly donors was characterized by cross-reactive (CR) antibodies recognizing non-HA antigens. Surprisingly, a substantial fraction of these CR antibodies targeted sulfated glycans typical of egg-produced proteins, which does not provide protection against human influenza viruses. Overall, these findings are of great value in understanding suboptimal immunity after influenza vaccination and shaping future vaccine efforts that will achieve increased protection in the elderly.
\end{abstract}

\section{Age-related characteristics} of post-vaccination antibody responses

Although seasonal vaccination has contributed substantially to reducing the number of hospitalizations and deaths due to influenza infections, the limited efficacy of influenza vaccines in the high-risk population, such as the elderly, still represents a major concern for public health (1). In fact, just for the 2018-2019 flu season in the United States, more than 25,500 deaths were reported among older adults (65 years or older), accounting for $75 \%$ of the total influenza-associated deaths. Alarmingly, the influenza mortality rate for this age group (approximately 49 per 100,000 population) was 24 times higher than the mortality rate for the young population (approximately 2 per 100,000 population) during the same period (2). With these estimates, it is evident not only that the elderly are highly vulnerable to influenza infections but also that current protective measures are not equally effective among the different age groups. Consequently, the study and understanding of potential signatures associated with diminished vaccine protection are crucial to developing more effective immunotherapeutic countermeasures, especially for the aging population.

During naturally occurring infections, the humoral response against influenza is mediated by antibodies that target the viral surface proteins hemagglutinin (HA), neuraminidase (NA), and, to a lesser extent, matrix protein 2 (M2). As the HA protein is critical for virus entry into the host cell, HA-specific antibodies, spe-

Related Article: https://doi.org/10.1172/JCI148763

Conflict of interest: The authors have declared that no conflict of interest exists.

cifically targeting the protein's receptorbinding site, are the most effective response to neutralize influenza viruses and prevent the disease (3). Consequently, successful influenza vaccines are expected to produce high, sustained HA inhibition antibody titers, even though HA antibodies targeting other epitopes or non-HA antibodies can also modulate the resulting disease $(4,5)$. As humoral immunity constitutes the main protection mechanism conferred by influenza vaccines, characterization of the serological repertoire of individuals in different age groups after vaccination is critical to identify antibody signatures differentiating aged individuals from younger subjects. In this context, it has been demonstrated that older adults produce fewer high-affinity antibodies, probably because of a decreased ability of their immune system to carry out somatic hypermutations and isotype switching. (6). Complementary findings furthermore indicate that one of the primary weaknesses of the elderly immune response is a diminished quantity of antibodysecreting plasmablasts rather than a reduced number of antibodies secreted per cell (7). On the other hand, it has also been found that, even though influenza vaccination could activate memory B cells with highly mutated Ig variable (V) genes in both the young and elderly, only younger individuals have been found to accumulate de novo somatic mutations. In contrast, older adults appear to have a fixed $B$ cell repertoire and a vaccine response that is likely dominated by a B cell recall instead of intraclonal diversification (8). Consequently, it is believed that the low adaptability of the antibody response in elderly individuals could lead to the activation of B cells that target highly conserved but low-immunogenic epitopes (8).

To further understand the molecular features of serological immunity to influenza in young and elderly individu- 

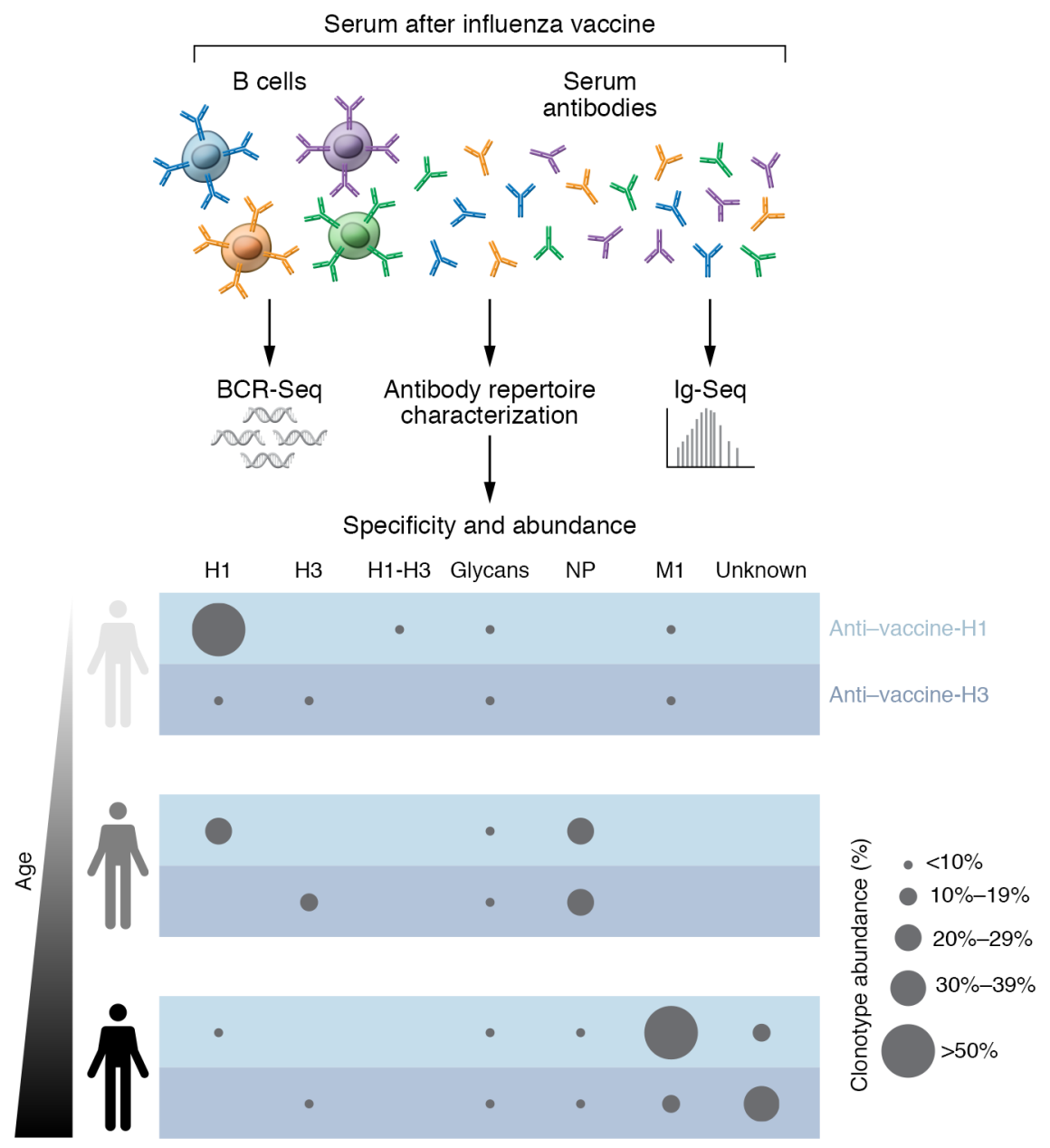

Figure 1. Analysis pipeline of serological repertoires after vaccination with a split, inactivated egg-based influenza vaccine. As a functional antibody response consists of BCRs expressed on the surface of B cells as well as of circulating antibodies in the blood, the analysis of both antibody repertoires is needed to study the humoral response generated after vaccination. Using BCR-Seq coupled with proteomic analysis of purified serum antibodies (Ig-Seq), Jung et al. found that, while the IgC clonotypic composition of young donors was predominantly specific for the $\mathrm{H} 1 \mathrm{or} \mathrm{H} 3$ components of the influenza vaccine, the clonotypic composition in older adults was dominated by CR antibody clonotypes. The binding specificity of a set of expressed antibodies further evidenced that young individuals had an $\mathrm{HA}$-focused response, whereas the response in older adults primarily targeted non-HA antigens, such as NP, M1, and egg-produced glycans. The bottom panel shows representative examples of the antibody specificity of serum antibodies according to Jung et al. (9).

als, in a recent issue of the JCI, Jung et al. performed a comprehensive analysis of the serum IgG repertoire after vaccination with a split, inactivated egg-based influenza vaccine (9) (Figure 1). Using high-throughput sequencing of transcripts encoding B cell receptors (BCR-Seq) along with proteomic analysis of antibodies in the blood (Ig-Seq), this research team found that cross-reactive (CR) antibody clonotypes dominated the IgG clonotypic composition of older adults. Interestingly, although young individuals' clonotypes are predominantly specific for the $\mathrm{H} 1$ or $\mathrm{H} 3$ components of the vaccine, immuniza- tion with the same vaccine in the following seasons promoted the expansion of $\mathrm{CR}$ clonotypes (Figure 1) (9). As older individuals have probably received more influenza vaccines than younger adults, it raises the question of whether the increase in $\mathrm{CR}$ clonotypes is a marker associated with age or whether it is a signature of repeated vaccinations reflecting only the vaccination history of a patient.

\section{Binding specificity of serum antibodies}

Regarding antibody specificity, it has been demonstrated that young adults vac- cinated with trivalent seasonal influenza vaccines exhibit a serum response mainly dominated by HA-specific antibodies (10). In agreement, Jung et al. confirmed that young donors had an HA-focused response with few antibodies recognizing NA proteins. On the other hand, serum from older adults seemed to be saturated with $\mathrm{CR}$ antibodies that primarily targeted non-HA antigens (9). Remarkably, Jung et al. demonstrated that the elderly had a substantial increase in antibodies aimed at conserved internal proteins, such as nucleoprotein (NP) and M1 (9). Surprisingly, however, the authors found that a substantial fraction of antibodies also bound to egg-produced glycosylation patterns. Regarding HA-specific CR antibodies, Jung et al. corroborated that these antibodies displayed broader HA recognition in the elderly compared with those from the younger participants (8, 9 , 11). Interestingly, these findings might support the study by Henry et al. (8), which showed that a poor adapted B cell response in the elderly can elicit antibodies focused on conserved antigens.

Concerning influenza protection, although NP- and M1-specific antibodies have also been detected in natural infections, it is unclear what their function is in humans $(12,13)$. Therefore, given the high prevalence of these antibodies in the elderly population, influenza vaccines probably have poor efficacy because NP and M1 are not relevant for protecting the individual or modulating the severity of the infection. Through their detailed biochemical characterization of identified and individually expressed antibodies, Jung et al. have also shown that a marked fraction of these characterized CR antibodies bound with high affinity to sulfated glycans only present in egg-produced HAs, specifically sulfated type $2 \mathrm{~N}$-acetyllactosamine and its variations (9). Since these glycans are not naturally produced during influenza infections, it is expected that their anti-glycan antibodies do not contribute to the protection against influenza. In the end, it appears that influenza vaccines have limited efficacy in the elderly, because only a small portion of the antibody response is fighting the infection. However, more studies are needed to determine how much non-HA antibodies can actually weaken the overall immune response. 


\section{Final remarks}

By using cutting-edge high-throughput technologies, Jung et al. performed an integrated study of how the antibody response to influenza vaccination changes according to age. As it is well known that influenza vaccines, as well as other vaccines, become less effective as patients age, this study is highly relevant for designing strategies that can improve protection in the elderly. One of the most striking findings in this investigation is that the serum repertoire of older adults was dominated by antibodies that did not recognize the most potent immunogens of the virus. Instead, the immune response was directed toward conserved antigens (NP, M1, and sulfated glycans prevalent in eggs), whose antibodies might not confer protection against the infection. As the effect of these antibodies is ambiguous, it will be interesting to evaluate whether they can show protection in vivo. Additionally, similar studies using subunit vaccines could help to identify how the immune response in the elderly changes without unwanted antigens. Finally, given the apparent propensity of antibodies in older adults to target conserved epitopes, the development of influenza vaccines focusing on conserved regions such as the HA stem would greatly benefit this population. Overall, the work by Jung et al. provides important insights into potential limitations of the immune response in the elderly upon vaccination with a split, egg-based vaccine. The findings also provide a word of caution about how we produce vaccines.

\section{Acknowledgments}

KJG and EMS are supported by the Collaborative Influenza Vaccine Innovation Centers (CIVIC) contract by the National Institute of Allergy and Infectious Diseases (NIAID), a component of the NIH, Department of Health and Human Services (contract 75N93019C00052 and grant R01AI140245). EMS is also supported by NIH grant R21AI143399.

Address correspondence to: Eva M. Strauch, 240 W. Green Street, University of Georgia, Athens, Georgia 30602, USA. Phone: 706.542.7725; Email: estrauch@ uga.edu.

1. Rondy M, et al. Effectiveness of influenza vaccines in preventing severe influenza illness among adults: a systematic review and meta-analysis of test-negative design casecontrol studies. JInfect. 2017;75(5):381-394.

2. Centers for Disease Control and Prevention. 2018-2019 Flu Season Burden Estimates. https://www.cdc.gov/flu/about/burden/2018-2019.html. Accessed July 7, 2021.

3. Bahadoran A, et al. Immune responses to influenza virus and its correlation to age and inherited factors. Front Microbiol. 2016;7:1841.

4. Hannoun C, et al. Immunogenicity and protective efficacy of influenza vaccination. Virus Res. 2004;103(1-2):133-138.

5. Wong SS, Webby RJ. Traditional and new influenza vaccines. Clin Microbiol Rev. 2013;26(3):476-492.

6. Frasca D, et al. Intrinsic defects in B cell response to seasonal influenza vaccination in elderly humans. Vaccine. 2010;28(51):8077-8084.

7. Sasaki S, et al. Limited efficacy of inactivated influenza vaccine in elderly individuals is associated with decreased production of vaccine-specific antibodies. J Clin Invest. 2011;121(8):3109-3119.

8. Henry C, et al. Influenza virus vaccination elicits poorly adapted B cell responses in elderly individuals. Cell Host Microbe. 2019;25(3):357-366.

9. Jung J, et al. Influenza vaccination in the elderly boosts antibodies against conserved viral proteins and egg-produced glycans. J Clin Invest. 2021;131(13):148763.

10. Lee J, et al. Molecular-level analysis of the serum antibody repertoire in young adults before and after seasonal influenza vaccination. Nat Med. 2016;22(12):1456-1464.

11. Ju CH, et al. Plasmablast antibody repertoires in elderly influenza vaccine responders exhibit restricted diversity but increased breadth of binding across influenza strains. Clin Immunol. 2018;193:70-79.

12. Padilla-Quirarte HO, et al. Protective antibodies against influenza proteins. Front Immunol. 2019;10:1677.

13. Krammer F. The human antibody response to influenza A virus infection and vaccination. Nat Rev Immunol. 2019;19(6):383-397. 